\title{
The bright side of negative experience: Preexposure to negative image improves non-judging attitude in mindfulness meditation
}

\author{
Kyoshiro Sasaki, ${ }^{1,2,3 *}$ Fuminori Ono, ${ }^{4}$ and Yuki Yamada ${ }^{2}$
}

\begin{abstract}
Mindfulness is a state of attending to current experiences without judging them and can be achieved by a meditation exercise called mindfulness meditation. A previous study revealed that non-judging of inner experiences, which is a facet of mindfulness, is related to the suppression of negative emotion. For a deeper understanding of this process, the present study examined whether the exposure to negative stimuli modulates the effect of mindfulness meditation. In the experiment, 24 participants were randomly assigned to one of two emotional conditions (negative and neutral). They observed a sequence of negative or neutral images and then engaged in mindfulness meditation. They also answered the Five Facet Mindfulness Questionnaire before and after the meditation. The results showed that the score on the non-judging subscale increased in the participants who were exposed to the negative images. The improvement in the non-judging attitude was possibly due to the psychological function that helps to suppress negative emotion that was evoked by the previous event.
\end{abstract}

\section{INTRODUCTION}

Mindfulness has attracted substantial attention in the field of psychology. Mindfulness has three aspects (Eberth \& Sedlmeier, 2012; Frewen et al., 2011; Hafenbrack, Kinias, \& Barsade, 2014): state mindfulness, trait mindfulness, and mindfulness meditation. State mindfulness is a state of directing attention to current experiences without judging them (Kabat-Zinn, 1994). Trait mindfulness is the tendency to live with good mental health and well-being (Baer et al., 2006). Mindfulness meditation is an exercise, in which practitioners focus attention to the present experience (e.g., breathing) and wash out other unwanted thoughts. Thus, mindfulness meditation is said to contribute to trait mindfulness (Baer, 2003; Baer et al., 2008). The practice of mindfulness meditation improves our mental condition, and improvement of mindfulness brings various beneficial consequences for our quality of life. The aim of the present study was to develop the way to enhance mindfulness, and surprisingly we found negative experience helpful.

Several studies have debated whether trait mindfulness has multiple facets. Some studies showed that trait mindfulness consists of four intercorrelated facets (Feldman et al., 2007; Walach et al., 2006). Other studies revealed that one facet is enough to measure trait mindfulness (Brown \& Ryan, 2003;

\footnotetext{
${ }^{1}$ Faculty of Science and Engineering, Waseda University, 3-4-1, Ohkubo, Shinjuku, Tokyo, 169-8555, Japan

${ }^{2}$ Faculty of Arts and Science, Kyushu University, 744 Motooka, Nishi-ku, Fukuoka, 819-0395, Japan

${ }^{3}$ Japan Society for the Promotion of Science, 5-3-1 Kojimachi, Chiyodaku, Tokyo 102-0083, Japan

${ }^{4}$ Faculty of Education, Yamaguchi University, 1677-1 Yoshida, Yamaguchi-shi, Yamaguchi 753-8513, Japan

*Corresponding author. E-mail: kyoshiro0920@gmail.com
}

Brown \& Ryan, 2004). Baer and her colleagues (Baer et al., 2006) conducted a joint factor analysis of the measures used in the above studies and conceptualized that trait mindfulness has five facets: observing, describing, acting with awareness, non-judging, and non-reactivity. Observing is a state of directing attention to internal and external events (sensation, emotion, and so on). Describing refers to explaining these events with proper words. Acting with awareness refers to attending to ones current behavior. Non-judging is an attitude of not evaluating thoughts and emotion. Non-reactivity is a tendency to ignore thoughts and emotion. These five dimensions could be measured by the Five Facet Mindfulness Questionnaire (FFMQ) (Baer et al., 2006, 2008), which is suitable for investigating the details of mindfulness (for a review, see Sauer et al., 2013). In regard to the FFMQ, the Japanese version has been developed and validated (Sugiura et al., 2012).

Recent studies examined the effect of mindfulness meditation on our cognition and behavior. For example, meditation practice has been found to improve attentional performance (MacLean et al., 2010; Zeidan et al., 2010), cognitive flexibility (Moore \& Malinowski, 2009) and working memory capacity (Mrazek et al., 2013). Although the participants in most of these studies experienced meditation training for as long as 4 days, recent studies have shown that just a few minutes of meditation influenced cognitive performance such as time perception (Kramer, Weger, \& Sharma, 2013), inattentional blindness (Schofield, Creswell, \& Denson, 2015), and emotion regulation (Arch \& Craske, 2006). Therefore, it is believed that mindfulness exercise has a big impact on our mental processing.

Several studies also revealed the relationship between trait mindfulness and negative emotion (Giluk, 2009). Mindfulness exercise contributes to reduction in negative emotion (Fennell, Benau, \& Atchley, 2016; Kiken \& Shook, 2014). Similar to other previous studies (Baer et al., 2006; Reynolds, Consedine, \& McCambridge, 2013), Kiken and Shook (2014) also revealed that greater trait mindfulness was linked with lower negative emotion. Especially, Reynolds et al. (2013) demonstrated that people with high scores on the non-judging facet felt less negative emotion than did those with low non-judging. Non-judging people also effectively suppress desires for food (Alberts et al., 2010), drink (Murphy \& MacKillop, 2012; Ostafin \& Marlatt, 2008), and gambling (Lakey et al., 2007). These previous findings are consistent with the concept of non-judging attitude (Baer et al., 2006): The attitude of parrying the thought and feeling without judging them. Briefly, the non-judging attitude is important 
for emotion regulation, and improvement of this attitude is beneficial for suppressing negative emotion.

Considering that a higher level of trait mindfulness leads to reduction in negative emotion (Baer et al., 2006; Kiken \& Shook, 2014; Reynolds et al., 2013) and trait mindfulness was improved by mindfulness exercise (e.g., Baer, 2003; Baer et al., 2008), it is possible that a negative experience also modulates the effect of mindfulness meditation on trait mindfulness. This is because when people experience strongly negative events, there should be a psychological function that helps them to recover from mental shock. Such a function is known as psychological resilience (Ihaya et al., 2010), and mindfulness possibly contributes to this function. If this hypothesis is true, the non-judging attitude in trait mindfulness, which is involved with the suppression of negative emotion (Reynolds et al., 2013), would be improved in order to attenuate negative emotion after encountering negative events. To verify this hypothesis, we examined whether the preexposure to negative stimuli modulates the non-judging attitude in trait mindfulness.

\section{METHOD}

\section{A. Participants}

Twenty-five volunteers participated in the experiment (mean age $=21.25$ ). All were unaware of the purpose of the experiment and reported normal vision. Moreover, they had no practice of mindfulness meditation. The ethics committees of Kyushu University approved the protocol of the present study. The experiment was conducted according to the guidelines established by the Helsinki Declaration. We obtained written informed consent from all participants.

\section{B. Stimuli and Procedure}

The present test battery consisted of visual images and the Japanese version of the FFMQ (Sugiura et al., 2012), which consisted of 39 items ( 7 items on observing, 8 on describing, 8 on acting with awareness, 8 on non-judging, and 8 on non-reactivity). We selected 10 trypophobic and neutral images from the images used in Experiment 2 in Le et al.s study (Le, Cole, \& Wilkins, 2015). Trypophobic images are the ones that present a cluster of holes or holelike objects (e.g., a lotus seed pod). The neutral images presented a trumpet, golf cup, and so on. Previous studies showed that trypophobic images robustly induced negative emotion (Chaya et al., 2016; Cole \& Wilkins, 2013; Le et al., 2015; Sasaki et al., 2017), which was necessary in the present study. Therefore, we used 10 trypophobic images as negative stimuli that were confirmed to be robustly negative by these previous studies. All the items of the Japanese version of FFMQ were scored on a 5-point scale from 1 (not at all applicable) to 5 (highly applicable). These image stimuli and questionnaires were presented in a paper form.

Each participant was randomly assigned to one of two emotional conditions: 14 participated in the negative condition and the rest in the neutral condition. We concurrently conducted the experiment for all the participants. The experiment consisted of four blocks: the pre-questionnaire, evaluation, meditation, and post-questionnaire blocks. In the pre-questionnaire block, participants responded to the Japanese version of the FFMQ. Then, in the evaluation block, the participants observed the images and evaluated the degree of discomfort of the images using an 11-point scale ranging from -5 (strong discomfort) to +5 (strong comfort). There were two emotional conditions: negative and neutral. In the negative and neutral conditions, the participants were presented with a set of the negative and neutral stimuli, respectively. None of the participants spent an extremely short or long time on the evaluation block. After the evaluation block, the participants practiced a 5-min exercise on Mindfulness of body and breath (Williams \& Penman, 2011), in which they were instructed to focus their attention on the movement of the breath in the body and to count their breaths from one to ten, repeatedly (for details, see Williams \& Penman, 2011). The experimenter sat with the participants to ensure task compliance. This exercise was designed for individuals with no prior experience of mindfulness meditation and has been used in extensively in previous studies (Kramer et al., 2013; Erisman \& Roemer, 2010; Mirams et al., 2013; Segal, Williams, \& Teasdale, 2002). After engaging in mindfulness meditation, the participants again responded to the Japanese version of the FFMQ in the post-questionnaire block.

\section{Data Analysis}

We calculated the sum of the rating scores of discomfort for all the images for each participant and used this as discomfort index. The participants whose discomfort index was more and less than 3 SD from the mean were excluded. To confirm whether there was a significant difference in the discomfort index between the negative and neutral conditions, we used a two-tailed $t$-test. We reported the Cohen' s $d$ as the effect size. After analysis on the discomfort index, we computed the Cronbach' $s$ alpha for all the facets in the FFMQ at the pre- and post-tests assessments. After computing the Cronbachs alpha, we calculated the sum of all the items of each facet in the FFMQ for each participant and used them as the observing, describing, acting with awareness, non-judging, and non-reacting indices. We conducted two-way mixed analyses of variance on each index with test timing (pre- and post-test) as a within-participant factor and stimulus (negative and neutral) as a betweenparticipants factor. The reported effect sizes were partial eta squares $\left(\eta_{p}{ }^{2}\right)$. The significance level of all the tests was .05 .

\section{RESULTS}

One participant in the negative condition was excluded because her discomfort index was more than $3 \mathrm{SD}$ from the mean. A two-tailed t-test revealed that the discomfort index was significantly lower in the negative condition than in the neutral condition $[t(22)=3.66, p=.001$, Cohen' $\mathrm{s} d=1.53]$, indicating that, as expected, the images used in the negative condition induced strong discomfort as compared to those used in the neutral condition.

The alpha in all the facets in the FFMQ was over .77 except for that in the observing facet $(\alpha=.67)$ in the pre- 

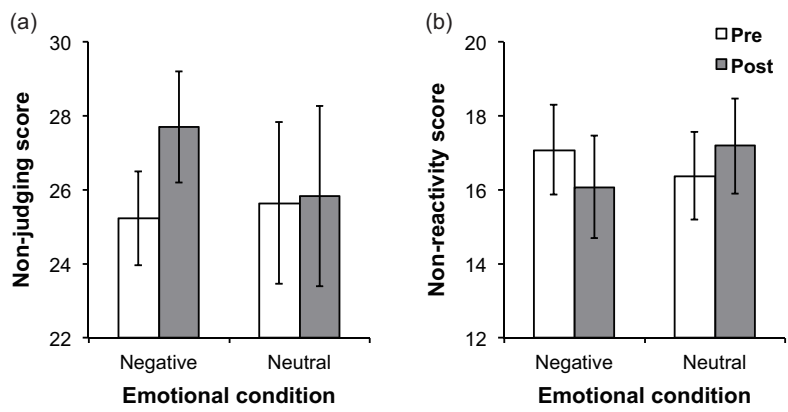

Fig. 1. The results of non-judging (a) and non-reacting (b) indices. Error bars denote the standard error of the mean.

test, while the alpha was over .73 on the all the facets in the post-test. We conducted two-way mixed ANOVAs on all the indices. On the observing, describing, and acting with awareness indices, there were no main effects or interactions [test timing: $F \mathrm{~s}(1,22)<1.74, p \mathrm{~s}>.20, \eta_{p}{ }^{2} \mathrm{~s}<.08$; stimulus: $F \mathrm{~s}(1,22)<0.94, p \mathrm{~s}>.33, \eta_{p}{ }^{2} \mathrm{~s}<.05$; interaction: $\left.F \mathrm{~s}(1,22)<1.60, p \mathrm{~s}>.21, \eta_{p}{ }^{2} \mathrm{~s}<.07\right]$. On the non-judging index (Figure 1a), the main effect of test timing and the interaction were significant $\left[F(1,22)=8.90, p=.007, \eta_{p}{ }^{2}\right.$ $=.29 ; F(1,22)=6.62, p=.017, \eta_{p}{ }^{2}=.23$, respectively $]$. The simple main effect of test timing was significant in the negative condition $\left[F(1,22)=15.44, p=.0007, \eta_{p}{ }^{2}=.41\right]$ but not significant in the neutral condition $[F(1,22)=0.08$, $\left.p=.77, \eta_{p}{ }^{2}=.003\right]$. On the non-reacting index (Figure 1b), the interaction was significant $\left[F(1,22)=5.18, p=.033, \eta_{p}{ }^{2}\right.$ $=.19]$. The simple main effect of test timing was marginally significant in the negative condition $[F(1,22)=3.14, p=$ $.09, \eta_{p}{ }^{2}=.12$ ] but not significant in the neutral condition $\left[F(1,22)=2.10, p=.16, \eta_{p}^{2}=.09\right]$.

\section{DISCUSSION}

The present study showed that the effect of mindfulness meditation on the non-judging responses was modulated after exposure to negative stimuli, while exposure to neutral stimuli did not induce any effect. Based on previous findings that indicated that non-judging contributed to the suppression of negative emotion (Reynolds et al., 2013), one plausible explanation of our results is that the non-judging attitude is improved in order to reduce the negative emotion evoked by the previous event. The results also showed that the mindfulness meditation tended to decrease non-reactivity after exposure to negative stimuli. This is possibly because emotional reaction was inevitably induced by negative stimuli (Bargh, 1997; Baumgartner et al., 2006; Dolan \& Vuilleumier, 2003), and our participants could not ignore the salience of the emotion. Thus, the participants non-reactivity decreased after exposure to the negative image.

With regard to another possible mechanism which the exposure to negative stimuli increase the non-judging responses, we propose that enhancement of the proprioceptive sensitivity due to trypophobic images boosts the effect of mindfulness meditation. It is generally said that the trypophobic images induce a discomfort reaction (Chaya et al., 2016;
Cole \& Wilkins, 2013; Le et al., 2015; Sasaki et al., 2017), which is an automatic reaction of the sympathetic nervous system known as horripilation. In addition to this, observing the trypophobic images induces skin crawling, shivering, and/or nausea (Chaya et al., 2016; Le et al., 2015). Here, we speculate that these discomfort bodily reaction might enhance attention to the self body (Mor \& Winquist, 2002), that is, proprioception (e.g., the awareness of limb positions, movements, and muscle tensions). On the other hand, Naranjo and Schmidt (Naranjo \& Schmidt, 2012) showed that mindfulness meditation improved practitioners motor control and increased their sensitivity to detect external perturbation, using a conflicting reaching task. They suggested that the mindfulness meditation leads to effective monitoring of ones body state (i.e., enhancement of proprioception). If the reverse is true, enhancement of the sensitivity of proprioception might affect the mindfulness attitudes. That is, in the present study increase of the non-judging responses was possibly due to the proprioceptive sensitization induced by the exposure to trypophobic images. Based on the current experimental design, we could not fully identify necessary factors about the proprioception to test this possibility, thus we leave it to future studies to determine the effect of proprioceptive manipulations on mindfulness meditation.

In the present study, we found that even a one-time, short meditation exercise was enough to promote non-judging in mindfulness after exposure to negative stimuli. Ordinarily, training programs over a certain period (e.g., mindfulnessbased stress reduction: Grossman et al., 2004) have been considered to be necessary for achieving mindfulness. In fact, the improvement of mindfulness did not occur after exposure to neutral stimuli in the present study. These findings indicate that exposure to negative emotion shortens the period of training and enhances the effectiveness of the mindfulness exercise. In future, clinical trials need to confirm whether exposure to negative stimuli is also helpful for training programs of mindfulness.

The modulation of the non-judging responses after the exposure to negative stimuli might support the neural underpinnings in emotional processing for trait mindfulness and mindfulness meditation. Previous studies have revealed that activity in the amygdala is reduced by repeated presentation of emotional stimuli (e.g., Wright et al., 2001) and that the decreased activation in the amygdala is important for emotion regulation (Beauregard, Levesque, \& Bourgouin, 2001; Harenski \& Hamann, 2006; Schaefer et al., 2002). Moreover, mindfulness meditation increased activity in the orbitofrontal cortex in meditation beginners, which is involved with reframing the evaluation of stimuli and with emotion regulation (Zeidan et al., 2011). Taken together, this suggests that activity in the amygdala and orbitofrontal cortex might decrease and increase, respectively, after exposure to negative stimuli and mindfulness meditation, thus enabling our participants (meditation beginners) to regulate their emotion efficiently. We speculate that this modulation of emotion regulation revealed in the present study leads to an increase in the nonjudging responses. In contrast to that observed in meditation 
beginners, meditation experts showed decreased activation in the dorsolateral and ventrolateral prefrontal cortices and the increased activation in the orbitofrontal cortex did not occur (Gard et al., 2012). This evidence from neuroscience suggests that exposure to negative stimuli activates different neural mechanisms in novice and expert meditators.

Although the present study revealed that the effect of mindfulness meditation on the non-judging responses was modulated by negative emotion, it is also likely that positive emotion has a similar effect. Several studies have showed the relationship between mindfulness and positive emotional experiences. For example, mindfulness meditation improves positive mood states (Jain et al., 2007). Moreover, previous studies revealed that high level of trait mindfulness was involved with various aspects of well-being (Baer et al., 2006; Brown \& Ryan, 2003; McCracken \& Yang, 2008). These healthy mental states, in turn, possibly contribute to promoting trait mindfulness, and as a result, a mindful life might be habitually maintained. Therefore, it is beneficial to examine the effect of exposure to positive emotion on mindfulness, compare this effect with the effect of negative emotion, and specify when exposure to each emotion is appropriate.

There are some limitations in the present study. First, we controlled the degree of exposure to negative events by the number of images, and thus it is unclear how long it took exposure to negative events to improve non-judging responses. The participants were exposed to negative stimuli only while they engaged in the evaluation task, and thus the amount of time spent in exposure to negative stimuli was approximately equal to the amount of time spent on the evaluation task. Here, to identify the approximately amount of time spent in exposure to negative stimuli, we asked another four participants to evaluate the discomfort of 10 images used in the experiment and measure time taken to complete the evaluation task. As a result, the mean elapsed time was 54.5 seconds ( $\mathrm{SD}=9.3$ ). Therefore, one-minute exposure is likely to be enough to alter non-judging responses. Second, one might wonder whether trait mindfulness was altered so fast within a short session. We used FFMQ that measured trait mindfulness, and hence it is plausible that trait mindfulness was improved in the present study. However, several previous studies have revealed that short exercises promoted state mindfulness (Kramer et al., 2013; Schofield et al., 2015; Arch \& Craske, 2006). It is possible that exposure to negative events and meditation exercise modulate state mindfulness, and this modulation is, in turn, involved with in our results. Further studies should address these issues.

\section{ACKNOWLEDGMENT}

The present study was supported by a Grant-in-Aid for JSPS Fellows (JP14J06025) given to K.S., a Grant-inAid for Challenging Exploratory Research (JP26540067) given to Y.Y., a Grant-in-Aid for Scientific Research (S) (JP15H05709) given to Y.Y., and Kyushu University Interdisciplinary Programs in Education and Projects in Research Development (26806 and 26307) given to Y.Y.
AVAILABILITY OF DATA AND MATERIALS

We submitted our data as follows: https://figshare.com/s/490d4534da09ba97eb87

\section{REFERENCES}

Alberts, H. J. E. M., Mulkens, S., Smeets, M., \& Thewissen, R. (2010) Coping with food cravings: investigating the potential of a mindfulness-based intervention. Appetite. $55,160163$.

Arch, J. J., \& Craske, M. G. (2006). Mechanisms of mindfulness: Emotion regulation following a focused breathing induction. Behaviour Research and Therapy, 44, 18491858.

Baer, R. A. (2003). Mindfulness training as a clinical intervention: A conceptual and empirical review. Clinical Psychology: Science and Practice, 10, 125143.

Baer, R. A., Smith, G. T., Hopkins, J., Krietemeyer, J., \& Toney, L. (2006). Using self-report assessment methods to explore facets of mindfulness. Assessment, 13, 2745.

Baer, R., Smith, G. T., Lykins, E., Button, D., Krietemeyer, J., Sauer, S., Walsh, E., Duggan, D., \& Williams, J. M. G. (2008). Construct validity of the five facet mindfulness questionnaire in meditating and nonmeditating samples. Assessment, 15, 329342.

Bargh, J. A. (1997). The automaticity of everyday life. In R. S. Wyer, Jr. (Ed.), The automaticity of everyday life: Advances in social cognition (Vol. 10, pp. 161). Mahwah, NJ: Erlbaum.

Baumgartner, T., Lutz, K., Schmidt, C. F., \& Jncke, L. (2006). The emotional power of music: How music enhances the feeling of affective pictures. Brain Research, 1075, 151164.

Beauregard, M., Levesque, J., \& Bourgouin, P. (2001). Neural correlates of conscious self-regulation of emotion. The Journal of Neuroscience, 21, 69937000.

Brown, K. W., \& Ryan, R. M. (2003). The benefits of being present: mindfulness and its role in psychological wellbeing. Journal of Personality and Social Psychology, 84, 822848.

Brown, K. W., \& Ryan, R. M. (2004). Perils and promise in defining and measuring mindfulness: observations from experience. Clinical Psychology: Science and Practice, $11,243248$.

Chaya, K., Xue, Y., Uto, Y., Yao, Q., \& Yamada, Y. (2016). Fear of eyes: Triadic relation among social anxiety, trypophobia, and discomfort for eye cluster. PeerJ, 4:e1942.

Cole, G. G., \& Wilkins, A. J. (2013). Fear of Holes. Psychological Science, 24, 19801985. Dolan, R. J., \& Vuilleumier, P. (2003). Amygdala automaticity in emotional processing. Annals of the New York Academy of Sciences, 985, 348355.

Eberth, J., \& Sedlmeier, P. (2012). The Effects of Mindfulness Meditation: A Meta-Analysis. Mindfulness, 3, 174189.

Erisman, S. M., \& Roemer, L. (2010). A preliminary investigation of the effects of experimentally-induced mind- 
fulness on emotional responding to film clips. Emotion, 10,7282 .

Feldman, G., Hayes, A., Kumar, S., Greeson, J., \& Laurenceau, J.-P. (2007). Mindfulness and emotion regulation: the development and initial validation of the Cognitive and Affective Mindfulness Scale-Revised (CAMSR). Journal of Psychopathology and Behavioral Assessment, 29, 177190.

Fennell, A. B., Benau, E. M., \& Atchley, R. A. (2016). A single session of meditation reduces of physiological indices of anger in both experienced and novice meditators. Consciousness and Cognition, 40, 5466.

Frewen, P., Lundberg, E., MacKinley, J., \& Wrath, A. (2011). Assessment of Response to Mindfulness Meditation: Meditation Breath Attention Scores in Association with Subjective Measures of State and Trait Mindfulness and Difficulty Letting Go of Depressive Cognition. Mindfulness, 2, 254269.

Gard, T., Hlzel, B. K., Sack, A. T., Hempel, H., Lazar, S. W., Vaitl, D., \& Ott, U. (2012). Pain attenuation through mindfulness is associated with decreased cognitive control and increased sensory processing in the brain. Cerebral Cortex, 22, 26922702.

Giluk, T. L. (2009). Mindfulness, big five personality, and affect: A meta-analysis. Personality and Individual Differences, 47, 805811.

Grossman, P., Niemann, L., Schmidt, S., \& Walach, H. (2004). Mindfulness-based stress reduction and health benefits. A meta-analysis. Journal of Psychosomatic Research, 57, 3543.

Hafenbrack, A. C., Kinias, Z., \& Barsade, S. G. (2014). Debiasing the mind through meditation: mindfulness and the sunk-cost bias. Psychological Science, 25, 369376.

Harenski, C.L., \& Hamann, S. (2006). Neural correlates of regulating negative emotions related to moral violations. NeuroImage, 30, 313324.

Ihaya, K., Yamada, Y., Kawabe, T., \& Nakamura, T. (2010). Implicit processing of environmental resources in psychological resilience. Psychologia, 53, 102113.

Jain, S., Shapiro, S. L., Swanick, S., Roesch, S. C., Mills, P. J., Bell, I., \& Schwartz, G. E. (2007). A randomized controlled trial of mindfulness meditation versus relaxation training: effects on distress, positive states of mind, rumination, and distraction. Annals of Behavioral Medicine, 33, 1121.

Kabat-Zinn, J. (1994). Wherever you go, there you are: mindfulness meditation in everyday life. New York: Hyperion.

Kiken, L. G., \& Shook, N. J. (2014). Does mindfulness attenuate thoughts emphasizing negativity, but not positivity? Journal of Research in Personality, 53, 2230.

Kramer, R. S. S., Weger, U. W., \& Sharma, D. (2013). The effect of mindfulness meditation on time perception. Consciousness and Cognition, 22, 846852.

Lakey, C. E., Campbell, W. K., Brown, K. W., \& Goodie, A. S. (2007). Dispositional mindfulness as a predictor of the severity of gambling outcomes. Personality and Individual Differences,43, 16981710.
Le, A. T. D., Cole, G. G., \& Wilkins, A. J. (2015). Assessment of trypophobia and an analysis of its visual precipitation. The Quarterly Journal of Experimental Psychology, 68, 119.

MacLean, K. A., Ferrer, E., Aichele, S. R., Bridwell, D. A., Zanesco, A. P., Jacobs, T. L., King, B. G., Rosenberg, E. L., Sahdra, B. K., Shaver, P. R., Wallace, B. A., Mangun, G. R., \& Saron, C. D. (2010). Intensive meditation training improves perceptual discrimination and sustained attention. Psychological Science, 21, 829839.

McCracken, L. M., \& Yang, S. Y. (2008). A contextual cognitive-behavioral analysis of rehabilitation workers' health and well-being: Influences of acceptance, mindfulness, and values-based action. Rehabilitation Psychology, 53,479485 .

Mirams, L., Poliakoff, E., Brown, R. J., \& Lloyd, D. M. (2013). Brief body-scan meditation practice improves somatosensory perceptual decision making. Consciousness and Cognition, 22, 348359.

Moore, A., \& Malinowski, P. (2009). Meditation, mindfulness and cognitive flexibility. Consciousness and Cognition, 18, 176186.

Mrazek, M. D., Franklin, M. S., Phillips, D. T., Baird, B., \& Schooler, J. W. (2013). Mindfulness training improves working memory capacity and GRE performance while reducing mind wandering. Psychological Science, 24, 776781.

Murphy, C., \& MacKillop, J. (2012). Living in the here and now: interrelationships between impulsivity, mindfulness, and alcohol misuse. Psychopharmacology, 219, 527536.

Naranjo, J. R., \& Schmidt, S. (2012). Is it me or not me? Modulation of perceptual-motor awareness and visuomotor performance by mindfulness meditation. $B M C$ Neuroscience, 13, 88.

Ostafin, B. D., \& Marlatt, G. A. (2008). Surfing the urge: Experiential acceptance moderates the relation between automatic alcohol motivation and hazardous drinking. Journal of Social and Clinical Psychology, 27, 404418.

Reynolds, L. M., Consedine, N. S., \& McCambridge, S. (2013). Mindfulness and disgust in colorectal cancer scenarios: Non-judging and non-reacting components predict avoidance when it makes sense. Mindfulness, 5, 442452.

Sasaki, K.*, Yamada, Y.* Kuroki, D., \& Miura, K. (2017). Trypophobic discomfort is spatial-frequency dependent. Advances in Cognitive Psychology, 13, 224-231. (*equal contribution)

Sauer, S., Walach, H., Schmidt, S., Hinterberger, T., Lynch, S., Bssing, A., \& Kohls, N. (2013). Assessment of mindfulness: Review on state of the art. Mindfulness, 4, 317.

Schaefer, S.M., Jackson, D.C., Davidson, R.J., Aguirre, G.K., Kim- berg, D.Y., \& Thompson-Schill, S.L. (2002). Modulation of amygdalar activity by the conscious regulation of negative emotion. Journal of Cognitive Neuroscience, $14,913921$. 
Schofield, T. P., Creswell, J. D., \& Denson, T. F. (2015). Brief mindfulness induction reduces inattentional blindness. Consciousness and Cognition, 37, 6370.

Segal, Z. V., Williams, J. M. G., \& Teasdale, J. D. (2002). Mindfulness-based cognitive therapy for depression: A new approach for preventing relapse. New York: Guilford Press.

Sugiura, Y., Sato, A., Ito, Y., \& Murakami, H. (2012). Development and validation of the Japanese version of the five facet mindfulness questionnaire. Mindfulness, 3 , 8594.

Walach, H., Buchheld, N., Buttenmuller, V., Kleinknecht, N., \& Schmidt, S. (2006). Measuring mindfulness; The Freiburg Mindfulness Inventory (FMI). Personality and Individual Differences, 40, 15431555.

Williams, M., \& Penman, D. (2011). Mindfulness: An eightweek plan for finding peace in a frantic world. Oxford: Piatkus.

Wright, C. I., Fischer, H., Whalen, P. J., McInerney, S. C., Shin, L. M., \& Rauch, S. L. (2001). Differential prefrontal cortex and amygdala habituation to repeatedly presented emotional stimuli. NeuroReport, 12, 379383.

Zeidan, F., Johnson, S. K., Diamond, B. J., David, Z., \& Goolkasian, P. (2010). Mindfulness meditation improves cognition: Evidence of brief mental training. Consciousness and Cognition, 19, 597605.

Zeidan, F., Martucci, K. T., Kraft, R. A., Gordon, N. S., McHaffie, J. G., \& Coghill, R. C. (2011). Brain mechanisms supporting the modulation of pain by mindfulness meditation. The Journal of Neuroscience, 31, 55405548. 